Ann. Zootech., r978, 27 (3), 423-437.

\title{
Solubilisation des protéines de poisson, supplémentation en tryptophane et valeur alimentaire pour le porcelet
}

\author{
B. SEVE, A. AUMAITRE, P. JAUberT $\left(^{*}\right)$ et P. TORD $\left(^{*}\right)$
}

avec la collaboration technique de Anne-Marie Mounier et A. LAPANOUSE

\author{
Station de Recherches sur l'Élevage des Porcs, \\ Centre national de Recherches zootechniques, I.N.R.A., \\ 78350 Jouy-en-Josas (France) \\ $\left(^{*}\right)$ Coopérative de traitement des produits de la pêche, \\ B.P. $36 I / 2,62203$ Boulogne-sur-Mer (France)
}

\section{Résumé}

L'hydrolyse enzymatique des déchets de poisson blanc permet d'obtenir des concentrés de protéines de poisson solubles dans l'eau dont la valeur nutritive varie selon les conditions de préparation. En appliquant un temps d'hydrolyse $T$, avec une concentration d'enzyme $E$, on obtient un premier produit $\left(\mathrm{E}_{1}\right)$ dont $75 \mathrm{p}$. 100 des protéines sont hydrosolubles. La solubilité augmente très sensiblement (de 75 à 86 p. roo) lorsqu'on multiplie par 8 le temps d'hydrolyse et par I,5 la quantité d'enzyme (produit $\mathrm{E}_{2}$ ). Un même taux de solubilité est obtenu avec un temps $2 \mathrm{~T}$ et une concentration enzymatique $\mathrm{I}, 25 \mathrm{E}$, si on élimine par centrifugation (" débourbage ") une partie du résidu non solubilisé (produit $\mathrm{E}_{3}$ ). La teneur en thréonine des protéines, exprimée en pourcentage de la somme corrigée des acides aminés indispensables $(\Sigma)$ augmente avec le taux de solubilité tandis que diminuent les taux d'histidine et de tryptophane. Ce dernier acide aminé apparaît nettement comme le facteur limitant primaire des protéines solubles de poisson ( $\mathbf{E}_{1}$ : Try $=0,0198 \Sigma ; E_{2}:$ Try $=0,0182 \Sigma ; E_{3}:$ Try $=0$,oI $\left.8 \mathrm{I} \Sigma\right)$. Au cours des essais de production industrielle, l'appareillage de l'usine a été adapté de telle sorte que la fabrication industrielle de Concentrés de Protéines Solubles de Poisson ayant la solubilité souhaitée est possible sans accroissement du prix de revient $n$.

Ces trois produits sont introduits dans des aliments de sevrage des porcelets à io jours où ils apportent 85 p. roo des protéines, le complément étant fourni par le lait écrémé (aliment i er âge) ou par l'orge (aliment $2^{\mathrm{e}}$ âge).

Une supplémentation en tryptophane est effectuée dans le cas des deux produits les plus déficients $\left(\mathrm{E}_{2}\right.$ et $\left.\mathrm{E}_{3}\right)$. Un lot témoin reçoit des aliments dont $66 \mathrm{p}$. Ioo des protéines proviennent du poisson (produit $\mathrm{E}_{1}$ ) et $33 \mathrm{p}$ roo du lait écrémé (aliment ${ }^{\mathrm{er}}$ âge) et d'un mélange orge-tourteau de soja (aliments $2^{e}$ âge). Avec le produit le moins soluble $\left(\mathrm{E}_{1}\right)$, les performances sont significativement plus élevées que dans le cas des produits les plus solubles $\left(\mathrm{F}_{2} \mathrm{et}_{\mathrm{F}}\right)$ non supplémentés. La consommation d'aliment augmente en moyenne de $z_{4} \mathrm{p}$. Ioo avec la supplémentation en tryptophane des produits $\mathrm{E}_{2}$ et $\mathrm{E}_{3}$. Parallèlement, les performances zootechniques, gain de poids et indice de consommation, s'améliorent de $3^{8}$ et i $\mathrm{p}$. Ioo respectivement. L'augmentation de la solubilité des protéines (produits $\mathrm{E}_{2}$ et $\mathrm{E}_{3}$ ) permet d'améliorer légèrement leur utilisation digestive par le porcelet de $2 \mathrm{I}$ à 28 jours d'âge $(+3,5 \mathrm{p}$. 100$)$. Cependant, un risque de dégradation de l'état sanitaire ou de mauvaise adaptation du porcelet à un aliment $2^{\mathrm{C}}$ âge est à souligner. L'ensemble des résultats confirme le déficit en tryptophane des protéines d'hydrolysat de poisson et permet de recommander une attaque enzymatique de faible intensité et de courte durée dans le cas des concentrés destinés aux porcelets. 


\section{Introduction}

Avant l'âge de trois semaines, le porcelet n'ingère spontanément que de faibles quantités d'aliment sec (AUMAITRE et SALMON-LEGAGNEUR, I96r) même s'il est sevré (Smith et LuCAS, I956). Cette sous-alimentation entraîne une pause de la croissance imputable d'abord au déficit des nutriments azotés absorbés (SEVE et Aumaitre, I978). En réalité, l'une des causes d'insuffisance de l'appétit du porcelet est probablement sa capacité digestive limitée par le faible développement des organes (Starovoitov, I956; Vodovar, Flanzy et Françors, 1964) et de leurs sécrétions de liquides et d'enzymes (PEkas, Thomson et Hays, I966; Cranweis, 1977; Corring, Aumaitre et Durand, I978). Compte tenu du caractère prioritaire de la nutrition azotée, la recherche de protéines très digestibles, ou des traitements technologiques à leur appliquer, est donc nécessaire avant la formulation des aliments de sevrage précoce.

La pratique d'une protéolyse enzymatique des aliments, avant leur ingestion, permettrait selon certains auteurs d'augmenter l'utilisation digestive des protéines, par le jeune porcelet (Clark, I960) (KaSpar et Prôkop, I97I) (SEvE et Aumaitre, 1973). Cependant nous avons montré dans un travail antérieur (SEVE, AUMAITre, TORD, I975) que la valeur biologique de protéines de poisson solubilisées par voie enzymatique varie selon le processus de fabrication. Les performances des animaux (rats et porcelets) étaient alors réduites lorsqu'une fraction non solubilisée par l'hydrolyse enzymatique avait été soustraite du produit final. Selon des travaux de SRIPATHY et al., I963, une telle opération conduirait à la production de protéines pauvres en tryptophane. Toutefois, les efiets d'une variation de la durée de l'hydrolyse sur la composition en acides aminés du produit final et sa valeur alimentaire pour l'animal sont mal connus. Ils font donc l'objet du présent travail, et à cette occasion, on a tenté chez le porcelet sevré à to jours, de corriger par supplémentation un déficit éventuel des aliments expétimentaux en $L$ tryptophane.

\section{Matériel et méthodes}

\section{I. - Préparation des farines solubles}

A partir d'une même matière première, déchets de poisson frais broyés et cuits, trois procédés de fabrication sont appliqués. Ils se distinguent par les opérations suivantes (tabl. I). A une même température de travail, on fait varier la durée de l'hydrolyse et la concentration d'enzyme.

Le "débourbage " permet d'éliminer la fraction non solubilisée par l'hydrolyse $\left(\mathrm{F}_{3}\right)$. Après quoi, une deuxième centrifugation de déshuilage a lieu, puis le produit est séché par atomisation (procédé Spray).

L'analyse des produits finaux figure au tableau 2. L'estimation de la teneur en protéines vraies est réalisée par précipitation à l'alcool à $80 \mathrm{p}$. Ioo.

La solubilité vraie des protéines est déterminée après dissolution dans l'eau distillée, dans la proportion pondérale de $\mathrm{I} / \mathrm{I} 0$, par centrifugation (Io $\mathrm{mn}$ à $600 \mathrm{~g}$ ) puis dosage de l'azote dans le culot et le surnageant. Les matières grasses sont dosées 


\section{TABLEAU I}

Conditions de fabrication des produits testés Manufacturing conditions of the tested products

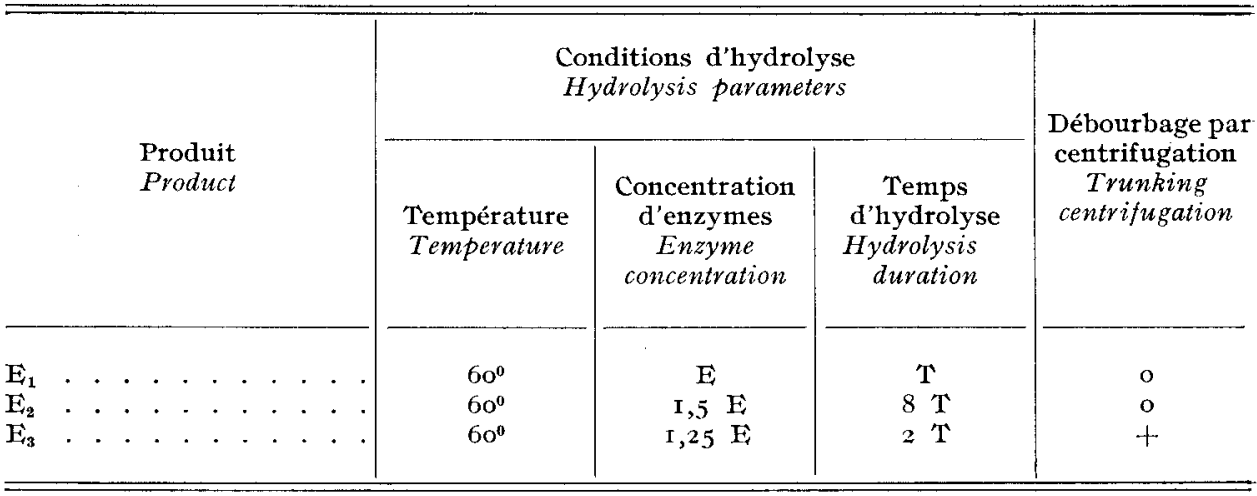

TABIEAU 2

Analyse des produits expérimentaux ( $p$. Ioo du produit frais)

Chemical composition of the products ( $p$. roo of air dry product)

\begin{tabular}{|c|c|c|c|}
\hline $\begin{array}{l}\text { Produits } \\
\text { Products }\end{array}$ & $\mathrm{F}_{1}$ & $\mathbf{E}_{2}$ & $\mathrm{E}_{3}$ \\
\hline Matiète sèche $($ Dry matter $) .. . . . . . . .$. & 97,5 & 97.5 & 97,5 \\
\hline Matières azotées totales (M.A.T) Crude protein (C.P.) & 83,2 & 83,7 & 84,3 \\
\hline - Protéines vraies (True protein). . . . . . & 32,5 & I9,6 & 25,6 \\
\hline - Protéines hydrosolubles (Soluble protein). & 75,0 & 87,0 & 86,0 \\
\hline Matières grasses totales (Total fat) . . . . & $\mathbf{1 0}, 8$ & 9,5 & II, 2 \\
\hline Matières minérales $(A s h) . . .$. & 6,8 & 6,7 & 5,6 \\
\hline
\end{tabular}

après hydrolyse en milieu chlorhydrique (3/4 d'heure à l'ébullition sous reflux), par extraction à l'éther éthylique $(6 \mathrm{~h})$. Les dosages d'acides aminés sont dus au. laboratoire de R. PION (communication personnelle) (tabl. 3).

\section{2. - Régimes expérimentaux}

Deux types d'aliments secs granulés $(\varnothing: 2,5 \mathrm{~mm})$ sans addition d'eau sont successivement distribués aux animaux. L'aliment $I^{\text {er }}$ âge renferme $22 \mathrm{p}$. Ioo de protéines et l'aliment $2^{\text {e }}$ age I 8 p. Ioo; ces deux taux sont volontairement suboptimaux de manière à mieux extérioriser les déséquilibres éventuels des protéines testées.

Six régimes expérimentaux sont constitués Les protéines du régime témoin (aliments $\mathrm{I}^{\text {er }}$ et $2^{\mathrm{e}}$ âge) proviennent pour $67 \mathrm{p}$. roo de la farine $\mathrm{E}_{1}$. Dans tous les 


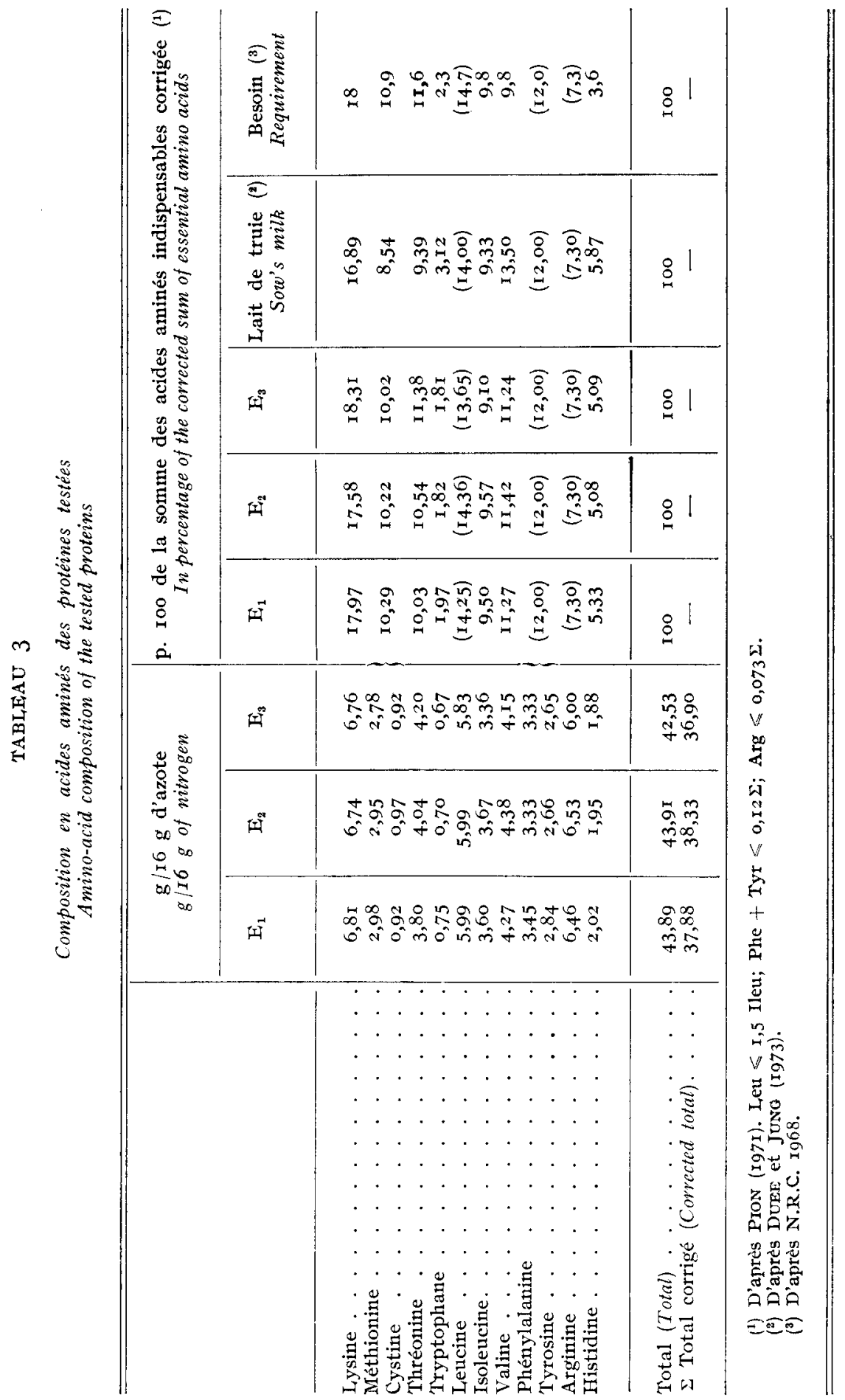


TABLEAU 4

Composition centésimale et analyse chimique des aliments expérimentaux I $^{\text {er }}$ age

Composition and chemical analysis of the prestarter feeds

\begin{tabular}{|c|c|c|c|c|c|c|}
\hline $\begin{array}{l}\text { Régime } \\
\text { Diet }\end{array}$ & $\begin{array}{l}\mathrm{I} \\
\text { Témoin } \\
\text { Control }\end{array}$ & 2 & 3 & 4 & 5 & 6 \\
\hline Produit testé (Tested product)...... & \multicolumn{2}{|c|}{$\mathbf{E}_{\mathbf{1}}$} & \multicolumn{2}{|c|}{$\mathrm{E}_{1 / 2}$} & \multicolumn{2}{|c|}{$\mathrm{E}_{3}$} \\
\hline 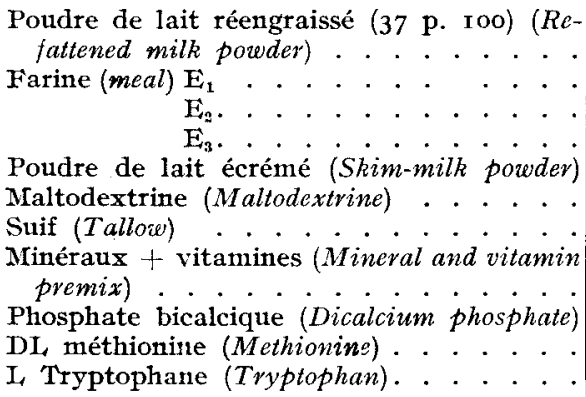 & $\begin{array}{r}18,80 \\
18,10 \\
- \\
12,10 \\
45,95 \\
3 \\
1,70 \\
0 \\
2,\end{array}$ & $\begin{array}{l}16,8 \\
23,4 \\
- \\
- \\
5^{2,25} \\
0^{2,20}\end{array}$ & 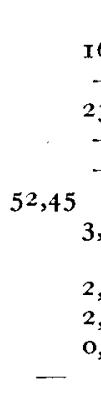 & $\begin{array}{l}, 7 \\
3 \\
52,4^{\circ} \\
0 \\
0 \\
0 \\
0,05\end{array}$ & 5 & $\begin{array}{l}7 \\
5^{\mathrm{I}, 70} \\
0 \\
0 \\
0 \\
0,05\end{array}$ \\
\hline 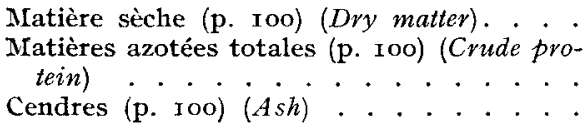 & $\begin{array}{r}93,4 \\
22,0 \\
4,6\end{array}$ & $\begin{array}{r}93,8 \\
21,9 \\
4,4\end{array}$ & $\begin{array}{r}93,1 \\
21,7 \\
4,3\end{array}$ & $\begin{array}{r}93,5 \\
21,9 \\
4,5\end{array}$ & $\begin{array}{r}9 z, 8 \\
21,5 \\
4,4\end{array}$ & $\begin{array}{r}92,9 \\
22,0 \\
4,2\end{array}$ \\
\hline 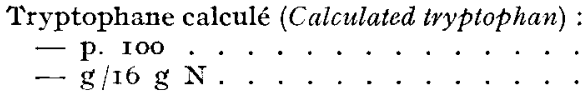 & $\begin{array}{l}0,194 \\
0,88\end{array}$ & $\begin{array}{l}0,178 \\
0,81\end{array}$ & $\begin{array}{l}0,169 \\
0,77\end{array}$ & $\begin{array}{l}0,219 \\
1,00\end{array}$ & $\begin{array}{l}0,163 \\
0,74\end{array}$ & $\begin{array}{l}0,213 \\
0,97\end{array}$ \\
\hline
\end{tabular}

autres aliments ( 2 à 6 ), les farines solubles de poisson fournissent $85 \mathrm{p}$. Ioo de l'apport azoté. Les trois produits $\mathrm{E}_{1} \mathrm{E}_{2}$ et $\mathrm{E}_{3}$ sont comparés dans les régimes 2,3 et 5 respectivement. Les effets de la supplémentation en tryptophane $(+0,05 \mathrm{p}$. IOo) des farines $\mathrm{E}_{2}$ et $\mathrm{E}_{3}$ sont testés dans les régimes 4 et 6.

Dans les aliments $I^{\text {er }}$ âge (tabl. 4) l'azote complémentaire provient du lait écrémé (33 et I 5 p. Ioo dans les aliments I et 2 à 6 respectivement). Le complément glucidique est fourni par une maltodextrine dérivant d'une hydrolyse partielle de l'amidon de maïs et très bien tolérée par le porcelet (SEvE, Aumaitre et Tord, I975).

En $2^{\mathrm{e}}$ âge (tabl. 5) les protéines complémentaires sont apportées par l'orge ( I5 p. IOo), associé à du tourteau de soja dans l'aliment témoin. Les glucides complémentaires proviennent du manioc que l'animal supporte en grande quantité dans sa ration dès l'âge de 5 semaines (AumaITre, I969).

A chaque stade les apports de matières grasses, de calcium et de phosphore sont sensiblement égalisés; il en est de même des teneurs en énergie brute, en lysine 


\section{TABLEAU 5}

Composition centésimale et analyse chimique des aliments expérimentaux $2^{\mathrm{e}}$ âge Composition and chemical analysis of the starter feeds

\begin{tabular}{|c|c|c|c|c|c|c|}
\hline $\begin{array}{c}\text { Régime } \\
\text { Diet }\end{array}$ & $\begin{array}{c}\text { I } \\
\text { Témoin } \\
\text { Control }\end{array}$ & 2 & 3 & 4 & 5 & 6 \\
\hline Produit testé (Tested product) & \multicolumn{2}{|c|}{$\mathrm{E}_{1}$} & \multicolumn{2}{|c|}{$\mathrm{E}_{12}$} & \multicolumn{2}{|c|}{$\mathrm{E}_{\mathbf{3}}$} \\
\hline $\begin{array}{l}\text { Farine (meal) } \mathrm{E}_{1} \\
\mathrm{E}_{2} \\
\mathrm{E}_{3}\end{array}$ & $\begin{array}{r}\frac{14,2}{-} \\
7,0 \\
26 \\
44,72 \\
3 \\
2 \\
2 \\
2 \\
2,08 \\
0,0 \\
2\end{array}$ & $\begin{array}{l}\text { I } 8,3 \\
\frac{-}{-} \\
44,85 \\
0 \\
0 \\
5 \\
0,05\end{array}$ & 478 & $\begin{array}{l}2 \\
5 \\
57,70 \\
0 \\
0 \\
5 \\
0,05\end{array}$ & $47,5^{2}$ & $\begin{array}{l}- \\
-1 \\
5 \\
5 \\
04,53 \\
0 \\
0 \\
5 \\
2 \\
12 \\
0,05\end{array}$ \\
\hline 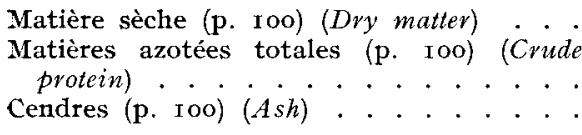 & $\begin{array}{r}91,6 \\
\mathbf{1} 8,0 \\
5,4\end{array}$ & $\begin{array}{r}92,2 \\
17,6 \\
5,6\end{array}$ & $\begin{array}{r}91,7 \\
17, \mathrm{I} \\
5, \mathrm{I}\end{array}$ & $\begin{array}{r}9 \mathrm{I}, 3 \\
\mathrm{I} 7,3 \\
5,6\end{array}$ & $\begin{array}{r}91,6 \\
\mathbf{1} 7,7 \\
5,4\end{array}$ & $\begin{array}{r}91,5 \\
18,0 \\
5,3\end{array}$ \\
\hline 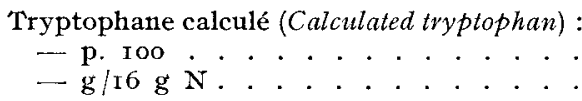 & $\begin{array}{l}0, \mathrm{I} 6 \mathrm{I} \\
0,89\end{array}$ & $\begin{array}{l}0, \mathrm{I} 45 \\
\mathrm{o}, 8 \mathrm{o}\end{array}$ & $\begin{array}{c}0, \mathbf{I} 37 \\
0,76\end{array}$ & $\begin{array}{l}0,187 \\
\mathrm{I}, 05\end{array}$ & $\begin{array}{l}0, \mathrm{I} 32 \\
0,73\end{array}$ & $\begin{array}{l}\mathrm{O}, \mathrm{I} 82 \\
\mathrm{I}, \mathrm{OI}\end{array}$ \\
\hline
\end{tabular}

et en acides aminés soufrés (supplémentation en DL méthionine). Les aliments non supplémentés sont légèrement déficients en tryptophane par rapport aux estimations minimales du besoin (N R C, I973).

\section{3. - Animaux. Schéma expérimental et conduite de l'expérience}

A dix jours d'âge, vingt portées de porcelets sont partagées chacune en trois couples équipondéraux formés chacun d'un mâle et d'une femelle. Chaque couple est affecté au hasard à l'un des six régimes ou traitements expérimentaux en application d'un plan d'expérience en blocs incomplets équilibrés de type 3 (CocHRAN et Cox, r957). Ce plan, complété avec ro portées, est répété deux fois. Au total, I 20 porcelets sont utilisés soit 20 par traitement expérimental.

De ro à 28 jours d'âge, les porcelets reçoivent les aliments rer âge seulement. Un remplacement progressif de ceux-ci par les aliments $2^{\mathrm{e}}$ âge est effectué de 28 à 42 jours d'âge. Au cours de cette première phase de l'expérience les animaux sont 
installés dans un local climatisé (1) en batterie d'élevage à trois étages, les couples d'une mème portée occupant des cases contiguës. Ils sont alimentés deux fois par jour en quantités ajustées à leur appétit et disposent d'eau de boisson à volonté (abreuvoir automatique). A partir de 6 semaines d'âge, ils sont transférés en porcherie classique sur sol paillé et reçoivent ad libitum, par couple, les aliments $2^{\mathbf{e}}$ âge en nourrisseur automátique et l'eau de boisson en abreuvoir de type siphoïde, jusqu'à l'âge de neuf semaines.

\section{4. - Mesures et observations}

Tous les aliments expérimentaux sort marqués par $0,3 \mathrm{p}$. Ioo d'oxyde de chrome en vue des estimations de l'utilisation digestive de leurs éléments. A partir de I4 jours d'âge, on effectue quotidiennement et par couple de porcelets des collectes d'échantillons fécaux. Ceux-ci sont regroupés en quatre périodes successives d'une semaine, jusqu'à 6 semaines d'âge des animaux.

Les porcelets sont pesés au sevrage, puis chaque semaine de I4 à 63 jours d'âge. Les quantités d'aliment consommé sont déterminées à chaque repas lors du séjour en batterie puis chaque semaine en porcherie. L'état sanitaire des animaux fait l'objet d'un enregistrement quotidien. Les troubles apparents et notamment les diarrhées sont traités si nécessaire par voie orale.

\section{Résultats}

\section{I. - Données analytiques (tabl. 2 et 3 )}

I a teneur en protéines vraies des matières azotées est fortement diminuée par l'allongement de la durée d'hydrolyse et l'addition d'enzymes au milieu, tandis que la solubilité augmente (produit $\mathrm{E}_{2}$ ). Cependant 1'opération de "débourbage " ( produit $\mathbf{E}_{3}$ ) entraîne une même variation de la solubilité accompagnée d'un abaissement plus modéré de la teneur en protéines vraies (tab1. 2).

Les résultats les plus remarquables concernent les modifications des teneurs en acides aminés essentiels (tabl. 3) exprimées notamment en pourcentage de la somme des A.A.I. corrigée selon PION (I97I). L'élimination partielle de la fraction non solubilisée entraîne une augmentation de la teneur en thréonine correspondant à une diminution des teneurs en tryptophane, isoleucine et histidine. Les effets de l'allongement du temps d'hydrolyse sont plus modérés et se traduisent par une augmentation du taux de thréonine et une diminution des teneurs en tryptophane et en histidine. Si on compare les profils à celui du lait de truie ou à un mélange équilibré des acides aminés essentiels calculé d'après l'estimation des besoins du porcelet (N.R.C., I g68) le tryptophane apparaît le facteur limitant primaire des protéines solubles de poisson. Les traitements visant à augmenter leur solubilité (produits $\mathrm{E}_{2}$ et $\mathrm{E}_{\mathrm{H}_{3}}$ ) tendent à accentuer le déficit de ce facteur limitant.

(1) T.air $=25^{\circ} \pm$ I; HR $=50$ p. I00 士 10 p. I00; v.air $<0$, IO $_{\mathrm{ms}^{-1}}$ 


\section{2. - Données zootechniques et nutritionnelles}

\section{a) Résultats zootechniques}

Nous avons considéré trois phases expérimentales dans la présentation des résultats : la période initiale (Io-28 jours) correspondant à la distribution exclusive des aliments I er âge, la période de séjour en batterie ( 10 à 42 jours) comprenant en plus de la phase précédente la transition avec l'aliment $2^{\mathrm{e}}$ âge, et en fin la période expérimentale complète (ro à 63 jours d'âge).

\section{Phase de démarrage (Io à 28 jours) (tabl. 6)}

Malgré la faiblesse du gain de poids, des différences entre régimes apparaissent nettement dès les premiers jours de l'expérience.

TABLEAU 6

Résultats de la période $I^{\mathrm{er}}$ âge (ro à 28 jours d'âge)

Results of the prestarter period ( $\mathrm{t} 0$ to 28 days of age)

\begin{tabular}{|c|c|c|c|c|c|c|c|}
\hline $\begin{array}{l}\text { Régime } \\
\text { liet }\end{array}$ & I & 2 & 3 & 4 & 5 & 6 & \\
\hline Produit testé (Tested produci). . . . & & $\mathbf{E}_{1}$ & & & & $i_{3}$ & \\
\hline $\begin{array}{l}\text { Taux de protéines de poisson (p. Ioo) } \\
\text { (level of fish protein)....... }\end{array}$ & 66 & 85 & & & & 35 & \\
\hline $\begin{array}{l}\text { Supplement de tryptophane (Extra } \\
\text { tryptophan) } . . \cdot \cdot \cdot . \cdot . \cdot .\end{array}$ & & o & 0 & + & 0 & $\therefore-$ & \\
\hline $\begin{array}{l}\text { Cain de poids (kg) (Weight gain) (1) } \\
\text { Aliment ingéré (kg) (Feed intake) (1) } \\
\text { Efficacité alimentaire (Gain jeed) (1) }\end{array}$ & $\begin{array}{l}\mathrm{I}, 44^{a} \\
3,02^{a} \\
0,4 \mathrm{I}^{a}\end{array}$ & $\begin{array}{l}1,22^{a b c} \\
2,95^{a b} \\
0,367^{a b}\end{array}$ & $\begin{array}{l}0,86^{d} \\
2,7^{b} \\
0,208 d\end{array}$ & $\begin{array}{l}\mathrm{I}, 34^{a b} \\
3,06^{a} \\
0,37^{a}\end{array}$ & $\begin{array}{l}0,92^{r t} \\
2,98 a b \\
0,279^{c}\end{array}$ & $\begin{array}{l}1,07^{b r d} \\
3,03^{a b} \\
0,3 \geq 0^{b c}\end{array}$ & $\begin{array}{l}s_{\bar{x}} \\
0,1 \mathrm{I} \\
0,09 \\
0,0 \mathrm{I} 7\end{array}$ \\
\hline
\end{tabular}

(1) I,es moyennes affectées d'exposants communs ne diffirent pas aul seuil 0,05 (Mcans reith same superscripts do not differ at the 0.05 level).

L'abaissement de 33 à $\mathbf{5}$ p. Ioo de la proportion des protéines de lait lorsqu'elles sont remplacées par la farine de solubilité minimale $\left(E_{1}\right)$ (lot 2$)$ n'entraîne pas de diminution significative du gain de poids, de la quantité d'aliment ingérée ni de l'efficacité alimentaire de $1 a$ ration. En revanche, 1'introduction des farines les plus solubles $\left(\mathrm{E}_{2}\right.$ et $\mathrm{E}_{3}$ ) conduit à un abaissement important de la vitesse de croissance et de l'efficacité alimentaire $(p<0,05)$ sans diminution significative du niveau d'ingestion d'aliment. I a supplémentation en tryptophane permet d'améliorer les trois critères étudiés $(p<0,05)$ dans le cas de l'aliment à base de farine $\mathrm{F}_{\mathrm{r}}$. 


\section{Phase de séjour des porcelets en batterie (ro à 42 jours) (tabl. 7 )}

Le remplacement du lait (lot $\mathrm{I}$ ) par les protéines de $\mathrm{E}_{1}$ (lot 2 ) entraîne une réduction significative de la vitesse de croissance; et l'introduction à forte dose des farines les plus solubles entraîne une nouvelle dégradation du gain de poids et de 1'indice de consommation. Cependant la supplémentation en tryptophane s'accompagne d'une stimulation de la consommation et se traduit par une amélioration hautement significative $(p<0,0 \mathrm{r})$ des performances de croissance $(32 \mathrm{p}$. IOo) et d'utilisation de la ration ( 18 p. IOo). Celles-ci avoisinent le niveau de celles obtenues avec le régime le plus riche en lait (lot I) dépassant ainsi les résultats obtenus avec la farine la moins soluble.

\section{TABLEAU 7}

Résultats généraux de l'expérience (périodes $10-42$ et $\mathrm{ro} 0-63$ jours) General vesults of the experiment

\begin{tabular}{|c|c|c|c|c|c|c|c|}
\hline $\begin{array}{l}\text { Régime } \\
\text { Diet }\end{array}$ & I & 2 & 3 & 4 & 5 & 6 & \\
\hline Produit testé (Tested product) & $\mathbf{E}$ & & & & & & \\
\hline $\begin{array}{l}\text { Taux de protéines de poisson (p. Ioo) } \\
\text { (Level of fish protein) }\end{array}$ & 66 & 85 & & & & & \\
\hline $\begin{array}{l}\text { Supplément de tryptophane (Extra } \\
\text { tryptophan) }\end{array}$ & 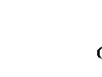 & & o & + & 0 & + & \\
\hline 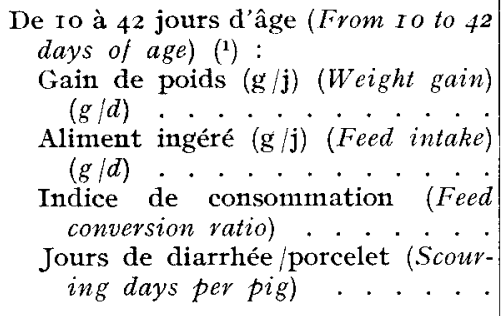 & $\begin{array}{l}\mathrm{II} 4^{a} \\
\mathrm{I} 57^{a b} \\
\mathrm{I}, 39^{a} \\
0,96^{a b}\end{array}$ & $\begin{array}{l}100^{b c} \\
149^{a b c} \\
\mathrm{I}, 55^{\mathrm{I}^{a}} \\
0,84^{a}\end{array}$ & $\begin{array}{r}86^{d} \\
\mathrm{I} 3^{8^{c}} \\
\mathbf{I}, 7^{\mathbf{I}^{b}} \\
\mathbf{I}, 3^{2^{a b}}\end{array}$ & $\begin{array}{l}\mathrm{II} 9^{a} \\
\mathrm{I} 6 \mathrm{I}^{a} \\
\mathrm{I}, 36^{a} \\
\mathrm{I}, \mathrm{I} 7^{a b}\end{array}$ & $\begin{array}{c}88^{c d} \\
\mathrm{I} 47^{b c} \\
\mathrm{I}, 74^{b} \\
0,9 I^{a b}\end{array}$ & $\begin{array}{l}111^{a b} \\
160^{a b} \\
\mathrm{I}, 4^{6} 6^{a} \\
\mathrm{I}, 87^{b}\end{array}$ & $\begin{array}{l}4,3 \\
4,4 \\
0,057 \\
-\end{array}$ \\
\hline 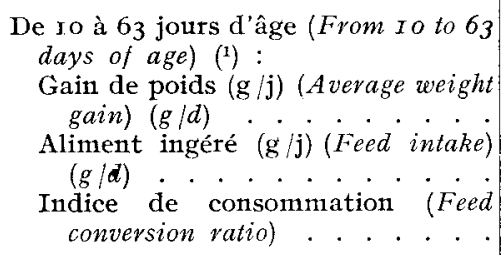 & $\begin{array}{l}25 \mathrm{I}^{a} \\
380^{a} \\
\mathrm{I}, 49^{a}\end{array}$ & $\begin{array}{l}207^{b} \\
326^{b} \\
I, 57^{a b}\end{array}$ & $\begin{array}{l}\mathrm{I} 72^{c} \\
27^{c} \\
\mathrm{I}, 65^{b c}\end{array}$ & $\begin{array}{l}224^{a b} \\
335^{b}\end{array}$ & $\begin{array}{l}59^{\circ} \\
274^{\circ}\end{array}$ & $\begin{array}{l}235^{a b} \\
35^{a b}\end{array}$ & $\begin{array}{l}\text { I I }, 0 \\
\text { I } 4,3\end{array}$ \\
\hline
\end{tabular}

(1) Les moyennes affectées d'exposants communs ne diffèrent pas atu seuil 0,05 (Means with same superscripts do not difer at the 0.05 level). 
Dans un contexte sanitaire généralement satisfaisant, la fréquence des diarrhées est plus élevée dans le lot 6 qui reçoit la farine $\mathrm{E}_{2}$ supplémentée. La différence avec le lot 2 qui reçoit la farine $\mathrm{E}_{1}$ est significative $(p<0,05)$.

\section{Période complète (Io à 63 jours) (tabl. 7)}

Les différences observées à 6 semaines d'âge ont tendance à s'accentuer au cours du séjour des porcelets en porcherie, alors qu'ils reçoivent l'aliment $2^{\mathrm{e}}$ âge. C'est ainsi que les effets des traitements permettant d'augmenter la solubilité $\left(\mathrm{E}_{2}\right.$ et $\left.\mathrm{F}_{3}\right)$ s'extériorisent définitivement par un abaissement significatif de la quántité d'aliment ingérée et du gain de poids accompagné d'une élévation de 1'indice de consommation $(p<0,05)$. La supplémentation en tryptophane permet dans les deux cas de compenser largement et de manière hautement significative cette dégradation des performances : en moyenne, la consommation d'aliment est augmentée de 24 p. Ioo, la vitesse de croissance de $38 \mathrm{p}$. Ioo, et l'efficacité alimentaire de II p. roo. Cependant, l'effet favorable du lait écrémé est confirmé par la supériorité du régime témoin, notamment sur le régime $n^{\circ} 2$ (farine $F_{1}$ ).

\section{TABLEAU 8}

Estimation de l'utilisation digestive apparente de la ration en fonction des modes de préparation des farines solubles testées Apparent digestibility of the diets

according to the manufacturing process of the soluble fish protein concentrates

\begin{tabular}{|c|c|c|c|c|c|c|c|}
\hline $\begin{array}{c}\text { Régime } \\
\text { Diet }\end{array}$ & I & 2 & 3 & 4 & 5 & 6 & \\
\hline Produit testé (Tested product) . . & & & & & & $z_{3}$ & \\
\hline $\begin{array}{l}\text { Taux de protéines de poisson (p. Ioo) } \\
\text { (Level of fish protein) }\end{array}$ & 66 & 85 & & & & 5 & \\
\hline $\begin{array}{c}\text { Supplément de tryptophane (Extra } \\
\text { tryptophan) }\end{array}$ & 0 & 0 & o & + & o & $\div$ & \\
\hline $\begin{array}{l}\text { CUDa (1) matière organique (Organic } \\
\text { matter): } \\
\begin{array}{l}\text { Période (Period }\left(^{2}\right): 2 \mathbf{I}-28 \text { jours } \\
28-35 \text { jours } \\
35-42 \text { jours }\end{array}\end{array}$ & $\begin{array}{l}92,7^{a b} \\
94,1^{a} \\
93,0^{a}\end{array}$ & $\begin{array}{l}92,3^{b} \\
93,4^{a b} \\
92,6^{a b}\end{array}$ & $\begin{array}{l}93,7^{a} \\
93,3^{a b} \\
93,1^{a}\end{array}$ & $\begin{array}{l}92,7^{a b} \\
92,4^{b} \\
92,0^{b}\end{array}$ & $\begin{array}{l}93,6^{a b} \\
93,6^{a b} \\
92,8^{a} b\end{array}$ & $\begin{array}{l}93,7^{a} \\
93,0^{a b} \\
92,5^{a b}\end{array}$ & $\begin{array}{l}s_{\bar{x}} \\
0,49 \\
0,50 \\
0,35\end{array}$ \\
\hline $\begin{array}{r}\text { CUDa (1) Azote (Nitrogen) : } \\
\text { Période }\left({ }^{2}\right) \text { (Period) }: 2 \mathrm{I}-28 \text { jours } \\
28-35 \text { jours } \\
35-4^{2} \text { jours }\end{array}$ & $\begin{array}{l}88,7^{a} \\
90,5^{a} \\
88,7^{a}\end{array}$ & $\begin{array}{l}86,3^{b} \\
88,4^{a b} \\
87,3^{a b}\end{array}$ & $\begin{array}{l}89,2^{a} \\
88,2^{a b} \\
88,4^{a}\end{array}$ & $\begin{array}{l}87,6^{a b} \\
86,1^{b} \\
85,7^{b}\end{array}$ & $\begin{array}{l}89,9^{a} \\
90,1^{a} \\
88,2^{a}\end{array}$ & $\begin{array}{l}89,7^{a} \\
88,2^{a b} \\
87,8^{a b}\end{array}$ & $\begin{array}{c}s_{x} \\
0,82 \\
0,82 \\
0,83\end{array}$ \\
\hline
\end{tabular}

(1) Coefficient d'utilisation digestive apparente (Apparent digestibility coefficient).

(3) A chaque période les moyennes affectées d'exposants communs ne diffèrent pas au seuil $0,05(A t$ each period, means with same superscripts do not differ at the 0.05 level). 
b) Résultats d'utilisation digestive des rations (tab1. 8)

Une variabilité très grande des résultats et notamment 1'hétérogénéité importante des variances compromettent l'interprétation des données de la Ire période (I4-2I jotirs).

A la seconde période ( 2 I à 28 jours), 1'utilisation digestive varie dans le même sens que la solubilité des protéines de la ration. La différence entre les régimes " $\mathrm{E}_{1}$ " et " $\mathrm{E}_{\mathbf{2}}$ » ou " $\mathrm{E}_{3}$ " est significative et plus marquée (3,5 p. Ioo en moyenne) dans le cas des protéines que dans celui de la matière organique. La supplémentation en tryptophane est sans effet sur l'utilisation digestive de la ration.

Au cours des deux périodes suivantes ( 28 à 42 jours d'âge) l'effet favorable d'une augmentation de la solubilité des protéines s'estompe avec l'introduction d'aliment $2^{e}$ agge dans la ration. On relève au contraire, dans le cas du régime 4 $\left(\mathrm{E}_{2}+\right.$ tryptophane) une baisse de l'utilisation digestive de la matière organique et des protéines qui s'accentue entre 35 et 42 jours d'âge. Les différences entre les lots $I$ et 4 sont significatives au seuil 0,05 .

\section{Discussion}

\section{I. - Données analytiques}

Cette expérimentation intéresse d'abord le domaine technologique et nos résultats permettent de préciser les effets de deux techniques d'augmentation de la solubilité des protéines de poisson sur les caractéristiques des concentrés obtenus.

I a diminution de la teneur en protéines vraies du produit avec l'augmentation du temips d'hydrolyse s'explique par une libération importante d'acides aminés dans le milieu au cours de la deuxième heure (SEN et al., I962). Par ailleurs, certains acides aminés apparaissent moins rapidement que d'autres dans la fraction soluble de 1'hydrolysat : il s'agirait de la phénylalanine, de l'isoleucine (HaLE, I969) et surtout du tryptophane (SRIPATHy et al., I963) (TARKY, AGarwalE et Pigot', I973). I1 n'est donc pas étonnant que ces acides aminés soient peu abondants dans la fraction solubilisée des déchets de poisson après une attaque enzymatique de courte durée $\left(\mathrm{E}_{3}\right)$.

En milieu pourtant légèrement acide $(\mathrm{pH}=5)$, il ne semble pas qu'une augmentation même considérable de la durée d'hydrolyse ( 2 à I7 h) entrâ̂ne la destruction d'une quantité significative de tryptophane (SRIPATHY et al., Ig63). Cependant, les parties osseuses riches en gélatine des poissons gadidés (SCHIL,ER et Schulz, r970) ne sont éliminées qu'après l'attaque enzymatique. Cette ci, en se prolongeant (produit $\mathrm{E}_{2}$ ) peut entraîner la dégradation d'une proportion plus importante de protéines dépourvues de tryptophane (gélatine) et expliquer ainsi l'appauvrissement du produit final en cet acide aminé.

\section{2. - Données zootechniques et nutritionnelles}

Les résultats de 1'essai peuvent être discutés en distinguant les effets de deux caractéristiques essentielles des farines testées, le déficit en tryptophane de leurs protéines et leur solubilité dans l'eau.

La supplémentation en L tryptophane des protéines de poisson les plus solubles 
$\left(\mathrm{E}_{2}\right.$ et $\left.\mathrm{E}_{3}\right)$ entraîne une augmentation du gain journalier de près de $30 \mathrm{p}$. Ioo alors que le niveau d'ingestion s'accroît de 24 p. Ioo. De même HENRY et PASTuszerwska (I976) soulignent l'effet dépressif d'une carence en tryptophane sur l'appétit en l'associant à la modification du profil des acides aminés libres sanguins. Dans nos conditions, chez des animaux plus jeunes très rationnés (zo à $30 \mathrm{~g}$ de matière sèche / $\mathrm{kg}$ de poids vif/jour), cet effet semble encore s'accentuer.

Le besoin en tryptophane du porc n'est pas connu avec précision. Une revue des données bibliographiques portant sur des stades variés de la croissance aboutit au chiffre moyen de $0,70 \mathrm{p}$. xoo des protéines de la ration (BOOMGAARDT et BAKER, 1973). En revanche, HENRY et PASTUSzewska (1976) ont montré récemment qu'un taux de $0,8 \mathrm{p}$. Ioo ne couvre pas le besoin des porcs en croissance. Chez le porcelet sevré précocement, l'imprécision est encore plus grande, GaLlo et POND (I966), puis LEWIs et al. (I977) observent qu'entre 5 et Io $\mathrm{kg}$ de poids vif les animaux répondent à une supplémentation jusqu'à un taux d'environ $I \mathrm{p}$. Ioo de tryptophane dans les protéines, alors que Zimmerman (I975) pense que le besoin est satisfait avec $0,8 \mathrm{p}$. Ioo. Ces divergences sont sans doute liées à la fois aux variations de la disponibilité et aux difficultés de dosage de cet acide aminé.

Nos données permettent en tous cas de confirmer que le besoin en tryptophane n'est pas couvert lorsque les protéines n'en renferment que 0,75 à 0,77 p. Ioo.

L'hydrolyse enzymatique des protéines de poisson, et notamment, l'emploi de la fraction la plus soluble de l'hydrolysat entraîne une légère amélioration de l'utilisation digestive de l'azote. Ce résultat confirme les données recueillies précédemment tant chez le porcelet (SEVE, AuMAITrE et TORD, I975) que chez le veau préruminant (TOULLEC, COROLLER et PATUREAU-MIrand, I977). Dans l'hypothèse où le besoin en tryptophane serait convert par le régime $2(0,80-0,8 \mathrm{I}$ p. Ioo) selon. ZIMMERMAN, I975, on pourrait attribuer au supplément de protéines digestibles la différence de vitesse de croissance observée entre les lots 2 et 4 ou 6 . En effet, le taux de protéines adopté dans l'essai est nettement suboptimal par rapport aux normes généralement retenues pour des porcelets aussi jeunes (ARC, I967; MüLLER et Kirchgessner, 1974). Nous avons montré par ailleurs (Seve et Aumaitre, I978) que toute augmentation de l'utilisation digestive de protéines se répercute sur le gain de poids des porcelets sevrés à Io jours. Cependant, dans l'expérience présente, l'absence d'un lot d'animaux recevant le produit $\mathrm{E}_{1}$ supplémenté en tryptophane ne permet pas de vérifier cette hypothèse.

L'emploi de protéines solubles de poisson pourrait donc contribuer à mieux satisfaire le besoin azoté du porcelet, cependant la distribution au porcelet de protéines excessivement solubles peut favoriser l'apparition de diarrhée (régime 6) ou compromettre l'adaptation ultérieure de l'animal à un aliment renfermant des céréales (régime 4) (Sfve, Aumaitre et 'Tord, I975). En plus de l'équilibre des acides aminés, il semble nécessaire d'apporter dans un rapport optimal des protéines natives et des protéines partiellement hydrolysées afin d'assurer le développement de la fonction digestive du porcelet sevré très précocement tout en satisfaisant son besoin azoté.

\section{Conclusion}

Les résultats de la présente étude confirment le déficit des protéines solubles de poisson en tryptophane. Deux facteurs de variation de ce déficit sont mis en évidence : en premier lieu, l'extraction de la fraction insoluble et en second lieu la durée de l'hydrolyse enzymatique. Sur le plan pratique, nos résultats permettent 
d'orienter le choix d'un processus technologique de production en fonction des objectifs recherchés, solubilité maximum ou équilibre optimum des protéines du produit final. Les processus technologiques utilisés permettent de réaliser industriellement les produits souhaités.

Dans le cas de l'alimentation azotée des porcelets sevrés précocément, l'intérêt d'augmenter la solubilité des protéines au-dessus d'un certain seuil n'apparaît pas clairement. Il semble au contraire qu'une attaque enzymatique de courte durée permette d'assurer la digestion d'une quantité suffisante de protéines par le porcelet sevré à Io jours tout en facilitant son adaptation à des aliments à base de céréales. Ainsi, la farine de solubilité minimale est très bien tolérée par l'animal sans supplémentation. Cependant, la vitesse de croissance maximale ne pourra être obtenue qu'en augmentant le taux de protéines de la ration au-dessus de 22 p. roo et surtout en maintenant la fraction fournie par le lait à 33 p. roo au moins, en accord avec nos résultats précédents (Seve, Aumaitre et Tord, I975; SEve, Février et Aumaitre, I975).

$$
\text { Accepté pour publication en mai } 1978 \text {. }
$$

\section{Remerciements}

Ce travail a bénéficié de l'aide de la D.G.R.S.T., dans le cadre de l'action coordonnée "Technologie Alimentaire et Agricole", Contrat n $^{\circ}$ 74-7-0o7.

\section{Summary}

\section{Solubilization of fish protein, tryptophan supplementation and nutritive value for the early weaned pigs}

Water-soluble fish protein concentrates (S.F.P.C.) can be obtained by enzyme hydrolysis of white fish by-products. A solubility of 75 p. roo was obtained for the first S.F.P.C. (E.) with a hydrolysis length $T$, and an enzyme concentration $E$ (Tables $I$ and 2 ). Solubility increased to $86 \mathrm{p}$. I oo when the hydrolysis lasted $8 \mathrm{~T}$ with an enzyme concentration of $\mathrm{I}, 5 \mathrm{E}$. A similar solubility $(87$ p. IOo) was observed with the combination $2 \mathbf{T}+\mathrm{r,25} \mathbf{E}$ when an important part of the unsolubilized fraction was centrifuged off. An increase in the threonine content together with a decrease in the histidine and tryptophan contents were found in $E_{2}$ and $E_{3}$ compared to $E_{1}$ (Table 3). This last amino-acid appeared to be the first limiting factor of the S.F.P.C.

The three products $\left(E_{1}, E_{2}\right.$ and $\left.E_{3}\right)$ supplied 85 p. Ioo of the crude protein in the experimental diets ( $\mathrm{n}^{\circ} 2,3$ and 5 ) of a trial involving 120 piglets weaned at 10 days and raised up to 63 days of age. The feeding schedule included two feeds, a prestarter containing $22 \mathrm{p}$. I oo protein and fed from $x$ to 42 days of age, a starter containing I 8 p. roo protein and fed from 28 to 63 days of age (Tables 4 and 5). The rest of the protein ( 5 p. roo) came from refattened skim-milk (prestarter)

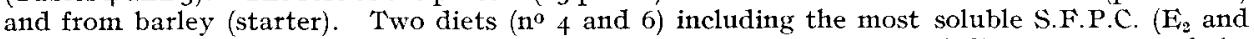
$\left.\mathrm{E}_{3}\right)$ were supplemented with $0.05 \mathrm{p}$. I oo $\mathrm{I}$ tryptophan. In the control diet, $67 \mathrm{p}$. I oo of the protein was supplied by S.F.P.C. $\mathrm{E}_{1}$ and 33 p. Ioo either by skim-milk in the prestarter feed or by a mixture of barley and soybean oilmeal in the starter feed. Performances, gain $(+$ I 8 p. Ioo) and feed efficiency $\left(+8 \mathrm{p}\right.$. IOo), were significantly higher with S.F.P.C. $E_{1}$ than with $E_{2}$ and $E_{3}$ in the unsupplemented diets (Table 7 ). Feed intake was enhanced $(+24$ p. roo) by the supplemental L tryptophan. In the same way, weight gain $\left(+3^{8} \mathrm{p}\right.$. Ioo) and feed efficiency $(+$ I I p. Ioo) were both improved. Digestibility of nitrogen was slightly $(+3,5 \mathrm{p}$. Ioo) but significantly increased from $2 \mathrm{I}$ to 28 days of age in the groups fed S.F.P.C. $\mathrm{E}_{2}$ and $\mathrm{E}_{3}$ compared to the experimental group fed S.F.P.C. $\mathbf{E}_{1}$ (Table 8). However, a tendency to more scouring and unsatisfactory adaptation to the starter feed was noted in some of those groups.

Tryptophan was finally confirmed to be the first limiting amino-acid of S.F.P.C. In preparing these concentrates for early weaned piglets, the enzyme treatment should be as mild as possible in order to facilitate protein digestion without degrading the biological value. 


\section{Références bibliographiques}

Agricultural Research Council, 1967. Nutrient requirements of farm livestock. No 3 , Pig, $278 \mathrm{p}$, A.R.C. ed., London.

Avmairre A., i969. Valeur alimentaire du manioc et de différentes céréales dans les régimes de sevrage précoce du porcelet. Ann. Zootech, 20, 55I-575.

Aumaitre A., Salmon Legagneur E., 196r. Influence de l'alimentation complémentaire de sevrage sur la croissance du porcelet avant le sevrage. Ann. Zootech, 10, I27-I 40.

BOOMGAARDT J., BAKER P. H., 1973. Tryptophan requirements of growing pigs at three levels of dietary protein. J. Anim. Sci, 36, 303-306.

ClARK A. J., 1960. Utilization of predigested soya protein by baby pigs. J. Anim. Sci., 19, 1254 (Abstr.).

Cochrax MI. G., Cox G. M., 1957. Experimental designs. and edition, 6II p., John Wiley and sons ed., New York.

Corring T., Aumaitre A., IUrand G., 1978. Development of digestive enzymes in the piglet from birth to 8 weeks. I. Pancreas and Pancreatic enzymes. Nutr. Mctab., 22, $23^{\mathrm{r}-243}$.

Crantelt, P. D., I977. Acid and pepsin secretion in young pigs reared solely by the sow or supplemented with solid food and weaned at 21 days. Proc. Nutr. Soc., 36, I 2 A

Duen P. H., Jung: J., I973. Composition en acides aminés du lait de truie. Ann. Zootech., 22, $243-247$.

Gallo J. T., Pond W. G., r966. Tryptophan requirement of early-weaned pigs from three to seven weeks of age. $J$. Anim. Sci., 25, $774-778$.

HALF M. B., I969. Relative activities of commercially available enzymes in the hydrolysis of fish protein. Food. Technol., 23, 107-t 10.

Henky Y., Pastuszewska B., 1976. Conséquences d'une déficience du régime en tryptoplrane chez le porc sur le niveau d'ingestion et les performances de croissance. Ann. Zootech., 25, I $43-148$.

KASPAR R., PROKOP V., 197I. Influencing of the digestibility of the nitrogenous substances of soy flour in the diet of early-weaned piglets (Tchèque). Zivocisna Vyroba (Praha), 16, 64 I-646.

Lewis A. H., Peo E. R. Jr., Crxisgham P. J., Mosfr B. D., 1977. Determination of the optimum dietary proportions of lysine and tryptophan for growing pigs based on growth, food intake and plasma metabolites. $J$. Nutr., 107, I 369-1 375

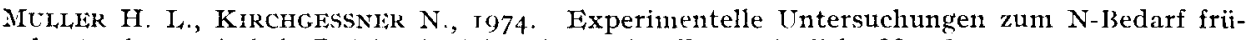
hentwöhnter Ferkel. Z. Ticrphysiol., Tievarnähr. Futtermittelkde, 33, 98-107.

National Research Council, I968. Nutrient requirements of domestic animals. $\mathrm{N}^{\circ} 2$, Nutrient requirements of swine, 6th revised edition, Nat. Acad. Sci., ed. publ., I599, Washington D.C.

National Research Council, r973. Nutrient requirements of domestic animals. No 2 , Nutrient requirements of swine, 7 th revised edition, Nat. Acad. Sci., ed. Washington D.C.

Pekas J. C., Thomson A. MI., Hays V. W., I966. Characteristics of the exocrine pancreatic secretion of the young pig. J. Anim. Sci, 25, II 3-12I.

Pion R., i97ı. Composition en acides aminés des aliments. Ind. Alim. Anim., 6, 29-36.

SCHIL.ER K., SCHULZ R., I97o. Der Einfluss und Herstellungs Verfahren auf die Inhaltstoffe und Qualität von Fischmehlen. Landwirtschaftliche Forschung., 23, I09-123.

Sex D. P., Sripathy N. V., Lahiky N. L., Srefinivasax A., Stbrahmaxyay V., ig6z. Fish hydrolysates. I. Rate of hydrolysis of fish with papain. Food Technol., 16, 138-141.

Seve B., Aumaitre: A., r973. Possibilités d'utilisation de concentrés de protéines solubles de poisson dans les aliments, d'allaitement artificiel pour porcelets. Journées Rech. Porcine en Fvance, Paris, I.N.R.A.-I.T.P. éd., 5, 95-104.

Seve B., Aumaitrt: A., Tord P., 1975. Valeur alimentaire de farines solubles de poisson blanc préparées selon divers procédés technologiques. Essais d'incorporation aux aliments d'allaitement artificiel du porcelet sevré à I 2 jours. Ann. Zootech., 24, 2 I-42.

SETE B., AUMaITrE A., $197^{8}$. Recent advances in protein nutrition of the early weaned pigs. World rev. anim. Prod., 14, 25-32.

Sevf B., Fevkifr C., Atmaitre A., I975. Alimentation glucidique du porcelet sevré à io jours. Effets du lactose, de l'ultrafiltrat de lactosérum et de la maltodextrine sur les performances du porcelet et l'utilisation digestive de la ration. Journées Rech. Porcine en France, Paris, I.N.R.A.-I.T.P. éd., 7, I0I-168. 
Smith H., LCCAS I. A. M., I956. The early weaning of pigs. I. The effect upon growth of variations in the protein, fat, sucrose, antibiotic, vitamin and mineral contents of diets for pigs of 8-25 rlb. weight and a comparison of wet and dry feeding. $J$. Agr. Sci., 48, 220-230.

Sripathy N. V., Kadkoi, S. B., Sen C. P., Swaminathan M., Lahiky N. L., 1963. Fish hydrolysates. III. Influence of degree of hydrolysis on nutritive value. J. Food. Sci, 28, 365-369.

Sitaxovoitov A. M., r956. Efficacité de la distribution de suppléments de céréales et d'aliments succulents aux porcelets non sevrés (Russe). Zhivotnovodstvo, 6, 46-5I.

Tarky W., Agarwale O. P., Pigott G. M., 1973. Protein hydrolysates from fish waste. $J$. Food. Sci., 38, 917-918.

Toullife R., Corollyk J. Y., Patureau Mirand P., i977. Utilisation des protéines par le veau préruminant à l'engrais. 6. Influence de la solubilité des protéines de maïs et de poisson sur leur utilisation digestive. Ann. Zootech., 26, 523-532.

Vodovar N., Flanzy J., François A. C., 1964. Intestin grêle du porc. I. Dimensions en fonction de l'âge et du poids de la jonction du canal cholédoque et du canal pancréatique à celui-ci. Ann. Biol. anim. Bioch. Biophys., 4, 27-34.

ZIMMERMAN D. R, I975. Tryptophan requirements of 5 to $15 \mathrm{~kg}$ pigs with semi-practical pig starters. J. Anim. Sci., 40, $875-879$. 